Schmerz 2014 · 28:125-127

DOI 10.1007/s00482-014-1418-2

Online publiziert: 9. April 2014

(c) Deutsche Schmerzgesellschaft e.V.

Published by Springer-Verlag Berlin Heidelberg - all rights reserved 2014
S. Förderreuther $\cdot A$. Straube

Klinik für Neurologie, Klinikum Großhadern, Ludwig-Maximilians-Universität München, München

\section{Kopfschmerztherapie - Schmerztherapie}

\section{Gemeinsamkeiten und Unterschiede}

Die vorliegende Ausgabe von Der Schmerz beschäftigt sich in drei Beiträgen aus verschiedenen Blickwinkeln mit chronischen Kopfschmerzen: Die Kieler Arbeitsgruppe diskutiert die Kriterien und die Therapie des Medikamentenübergebrauchskopfschmerzes (MÜK), Trotzeck u. Gaul greifen in einer Übersichtsarbeit die besondere Problematik auf, die sich mit dem Einsatz von Opiaten in der Behandlung von Kopfschmerzen einstellt. Und schließlich stellt die Deutsche Migräne- und Kopfschmerzgesellschaft (DMKG) in einem Positionspapier vor, welche Anforderungen an Kopfschmerzambulanzen und spezialisierte Kopfschmerzzentren bezüglich einer gestaffelten Regelversorgung von Kopfschmerzpatienten zu stellen sind.

Kopfschmerzen gehören mit einer 12 -Monats-Prävalenz von etwa $60 \%$ [16] zu den häufigsten Schmerzen überhaupt. Ungefähr 3\% leiden an chronischen Kopfschmerzen [19]. Chronischer Kopfschmerz ist durch sein Auftreten über mindestens 3 Monate an mehr als 15 Tagen im Monat über mindestens $4 \mathrm{~h}$ pro Tag definiert. Patienten mit chronischen Kopfschmerzen sind signifikant häufiger auch von Depression, Angsterkrankungen und Schmerzen anderer Lokalisation betroffen und bedürfen daher einer besonders intensiven Betreuung [2].

Ein großer Teil der Patienten mit chronischen Kopfschmerzen nimmt regelmäßig Schmerzmittel bzw. migränespezifische Medikamente ein (Triptane oder die heute nur selten eingesetzten Ergotamine, [17]), wobei die Prävalenz des MÜK in den Industrieländern mit 1-1,5\% angegeben wird [15]. Die Betaversion der dritten
International-Headache-Society(IHS)Klassifikation [7] führt bei den MÜK eine gravierende Änderung der Kriterien ein, die mit hoher Wahrscheinlichkeit zu einer Zunahme der Diagnose MÜK führen wird, da allein die Anamnese einer übermäßigen Akutmedikation (Triptane und Opiate an mehr als 10 Tagen, Analgetika an mehr als 15 Tagen) zusammen mit dem Auftreten von Kopfschmerzen an mehr als 15 Tagen zur Diagnose MÜK ausreicht.

In der vorherigen Version der IHSKlassifikation konnte die Diagnose erst gestellt werden, wenn es nach einer Medikamentenpause auch zu einer Besserung der Kopfschmerzen kam. Diese Änderung der Klassifikation ist der Tatsache geschuldet, dass sich nach einer Medikamentenpause nur bei etwa 50-70\% der Patienten eine Besserung einstellt, sodass bei den verbleibenden 30-50\% nach der alten Klassifikation kein Medikamentenübergebrauch diagnostiziert werden konnte [20].

Was die beiden Gruppen pathophysiologisch unterscheidet, ist nicht klar. Im Praxisalltag reicht bei vielen Patienten allein die Aufklärung über die Zusammenhänge zwischen Medikamenteneinnahme und Kopfschmerz, um eine nachhaltige Besserung einzuleiten. Andere Patienten dagegen erleiden trotz einer zunächst erfolgreichen Medikamentenpause multiple Rückfälle. Patienten mit psychischen Komorbiditäten und auch genetischer Belastung werden häufiger rückfällig als $\mathrm{Pa}$ tienten ohne diese Risikofaktoren [12] und brauchen auch eine engmaschigere Betreuung im weiteren Verlauf [9].

\section{Bedeutung der Prophylaxe}

Chronische Patienten und solche mit hohem Risiko einer Chronifizierung erfordern besondere Versorgungsstrukturen, die beispielsweise als unabdingbaren Bestandteil der Therapie die Schulung von Patienten zu ihrer Erkrankung ermöglichen und die Einleitung von medikamentösen und nichtmedikamentösen Therapien gewährleisten, die sich an den individuellen Besonderheiten des Patienten orientieren. Die Bedeutung von Aufklärung über die Folgen von Medikamentenübergebrauch konnte in einer epidemiologischen Studie aus Norwegen gezeigt werden [6]. Schon wenige Minuten Aufklärung über die Bedeutung eines Medikamentenübergebrauchs führten in einer populationsbezogenen epidemiologischen Studie zu einer Reduktion des MÜK um 76\%. Das Ergebnis mag überraschen, denn es zeigt, dass sich Einschätzung und Erwartung in der Medizin nicht immer decken. Eine italienische Studie konnte ebenfalls zeigen, dass bei leichter Betroffenen bezüglich des Therapieerfolgs kein Unterschied zwischen einem ambulanten, teilstationären und stationären Entzug bestand [10].

So ist auch die alte Lehrmeinung gefallen, eine Migräneprophylaxe sei bei Medikamentenübergebrauch unwirksam.

Diverse Studien haben dies überzeugend belegt $[3,13,18]$. Die Einleitung einer Prophylaxe für chronische Kopfschmerzpatienten ist ein zusätzlicher wichtiger 
Anker in der Behandlung. Eine Prophylaxe stellt eine zweite Säule der Therapie dar und schafft für die Patienten so zusätzliches Zutrauen in die Behandlung und Hoffnung für die Phase nach dem Entzug [9].

Die Lehrmeinung, dass Opiate in der Behandlung von Kopfschmerzen keinen Stellenwert haben, wird dagegen durch die Übersichtsarbeit von Trotzeck u. Gaul weiter gefestigt. Gerade der Gebrauch von Opiaten in der Akuttherapie von primären Kopfschmerzen ist mit einem signifikant erhöhten Risiko der Entwicklung von chronischen Kopfschmerzen vergesellschaftet [1]. Die europäischen Leitlinien vertreten diesen Standpunkt auch einheitlich im Gegensatz zu der Praxis in Amerika. Spannend wäre es, zu prüfen, ob auch bei nicht-tumorbedingten Schmerzen anderer Lokalisation ein Risiko der Chronifizierung unter einer Opiattherapie besteht. Entsprechende Arbeiten fehlen bislang.

Die Versorgung von Patienten mit hochfrequenten oder chronischen primären Kopfschmerzen hat sich in den letzten Jahren grundlegend gewandelt. Konzentrierte sich die Therapie noch vor 20 Jahren vorwiegend auf die pharmakologische Akuttherapie, so gewinnt die prophylaktische Therapie zunehmend an Bedeutung. Während diese initial ebenfalls primär pharmakologisch ausgerichtet war, z. B. mit $\beta$-Blockern, trizyklischen Antidepressiva oder Antikonvulsiva, wurden in den letzten Jahren biopsychosoziale Zusammenhänge zunehmend besser verstanden und in die Behandlungskonzepte integriert. Multimodale und interdisziplinäre Therapiekonzepte, die pharmakologische Therapien mit psychologischen Verfahren (Entspannungstherapie, Verhaltenstherapie, kognitive Verfahren, Biofeedback) und auch Sporttherapie kombinieren, gehören längst zum Standard der Kopfschmerzbehandlung und haben ihre Überlegenheit gegenüber einer rein pharmakologischen Therapie bewiesen [8].

Interdisziplinäre und multiprofessionelle Therapiekonzepte erfordern entsprechende Strukturen - dies gilt für die Kopfschmerzbehandlung so wie für die Behandlung anderer chronischer Schmerzerkrankungen. In den letzten 8 Jahren haben sich diesem Konzept fol- gend integrierte Versorgungszentren für Patienten mit Kopfschmerzerkrankungen etabliert [4], in denen nach strukturierten Vorgaben Therapiealgorithmen umgesetzt werden. Erste Evaluierungen der Therapieerfolge im Langzeitverlauf zeigen eine langfristige mindestens 50\%ige Besserung bei der Mehrzahl der so behandelten Patienten [5].

\section{Das DMKG-Stufenkonzept}

In der aktuellen Ausgabe von Der Schmerz schlägt die DMKG nun eine 3-stufig gegliederte Struktur für die Versorgung von Kopfschmerzpatienten vor. Dabei orientiert sich das Strukturpapier der DMKG mit den strukturellen Anforderungen, die jeweils an die Kopfschmerzeinrichtungen gestellt werden, an den Vorschlägen der European Headache Federation (EHF; [14]) und an den Kriterien, die von der Deutschen Schmerzgesellschaft zur Klassifikation schmerztherapeutischer Einrichtungen in Deutschland formuliert wurden [11]. Die therapeutischen Vorgaben sind dabei gut mit denen allgemeiner schmerztherapeutischer Zentren vergleichbar. Im Gegensatz zu den meisten anderen chronischen Schmerzerkrankungen, die grundsätzlich als symptomatisch einzuordnen sind, erfordert die Behandlung von Kopfschmerzen jedoch zusätzlich besondere diagnostische Voraussetzungen, da grundsätzlich die Unterscheidung zwischen primären und symptomatischen Formen erforderlich ist. Dies spiegelt sich in den Anforderungen an die besondere neurologische Expertise und die Möglichkeit zur entsprechenden Diagnostik wider. Eine grundsätzliche gesundheitspolitische Frage ist, inwieweit solche strukturellen Vorgaben in der Zeit von Budgetkürzungen umgesetzt werden können.

Die Diagnostik und Therapie von Kopfschmerzen ist in vielen, aber nicht allen Punkten mit der Therapie anderer chronischer Schmerzen vergleichbar. Gemeinsam sind häufige psychiatrische Komorbiditäten, die einer entsprechenden Therapie bedürfen, aber auch das Auftreten anderer chronischer Schmerzen. Die Pharmakotherapie unterscheidet sich wesentlich, da viele Nichtanalgetika zur Prophylaxe eingesetzt werden. Opia- te dagegen, die aus der Behandlung vieler Schmerzerkrankungen nicht wegzudenken sind, haben in der Kopfschmerztherapie nicht nur keinen Stellenwert, sie sind durch ihr hohes Potenzial zur Chronifizierung von Kopfschmerzen sogar schädlich. Zudem müssen bei Kopfschmerzerkrankungen anders als bei anderen chronischen Schmerzen sowohl zu Beginn als auch manchmal im weiteren Verlauf symptomatische Ursachen für den Kopfschmerz ausgeschlossen werden.

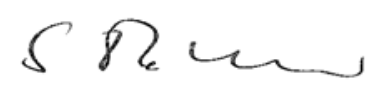

S. Förderreuther

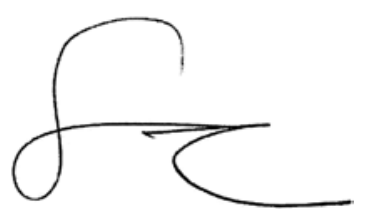

A. Straube

\section{Korrespondenzadresse}

Prof. Dr. A. Straube

Klinik für Neurologie, Klinikum Großhadern, Ludwig-Maximilians-Universität München

Marchioninistr. 15, 81377 München

andreas.straube@med.uni-muenchen.de

\section{Einhaltung ethischer Richtlinien}

Interessenkonflikt. S. Förderreuther und A. Straube geben an, dass kein Interessenkonflikt besteht.

\section{Literatur}

1. Bigal ME, Serrano D, Buse D et al (2008) Acute migraine medications and evolution from episodic to chronic migraine: a longitudinal population-based study. Headache 48:1157-1168

2. Bloudek LM, Stokes M, Buse DC et al (2011) Cost of healthcare for patients with migraine in five European countries: results from the International Burden of Migraine Study (IBMS). J Headache Pain;13:361-378

3. Diener HC, Bussone G, Van Oene JC et al (2007) Topiramate reduces headache days in chronic migraine: a randomized, double-blind, placebo-controlled study. TOPMAT-MIG-201(TOP-CHROME) Study Group. Cephalalgia;27:814-823

4. Diener HC, Gaul C, Jensen R et al (2011) Integrated headache care. Cephalalgia 31:1039-1047 
5. Gaul C, van Doorn C, Webering N et al (2011) Clinical outcome of a headache-specific multidisciplinary treatment program and adherence to treatment recommendations in a tertiary headache center: an observational study. J Headache Pain 12:475-483

6. Grande RB, Aaseth K, Benth JŠ et al (2011) Reduction in medication-overuse headache after short information. The Akershus study of chronic headache. Eur J Neurol 18:129-137

7. Headache Classification Committee of the International Headache Society (IHS) (2013) The International Classification of Headache Disorders, 3rd edition (beta version). Cephalalgia 33:629-808

8. Holroyd KA, Cottrell CK, O'Donnell FJ et al (2010) Effect of preventive (beta blocker) treatment, behavioural migraine management, or their combination on outcomes of optimised acute treatment in frequent migraine: randomised controlled trial. BMJ 341:C4871

9. Marziniak M, Haag G, May A et al (2013) Therapie des Kopfschmerzes bei Medikamentenübergebrauch. Evidenzbasierte Empfehlungen der DMKG, ÖSKG und SKG. Nervenheilkunde 32:135-144

10. Rossi P, Di Lorenzo C, Faroni J et al (2006) Advice alone vs. structured detoxification programmes for medication overuse headache: a prospective, randomized, open-label trial in transformed migraine patients with low medical needs. Cephalalgia 26:1097-1105

11. Sabatowski R, Maier C, Willweber-Strumpf A et al (2011) Empfehlungen zur Klassifikation schmerztherapeutischer Einrichtungen in Deutschland. Schmerz 25:368-370, 372-376

12. Saper JR, Hamel RL, Lake AE III (2005) Medication overuse headache $(\mathrm{MOH})$ is a biobehavioral disorder. Cephalalgia 25:545

13. Silberstein SD, Blumenfeld AM, Cady RK et al (2013) OnabotulinumtoxinA for treatment of chronic migraine: PREEMPT 24-week pooled subgroup analysis of patients who had acute headache medication overuse at baseline. J Neurol Sci 331:4856

14. Steiner TJ, Antonaci F, Jensen R et al (2011) Recommendations for headache service organization and delivery in Europe. J Headache Pain 12:419-426

15. Straube A, Pfaffenrath V, Ladwig KH et al (2010) Prevalence of chronic migraine and medication overuse headache in Germany - the German DMKG headache study. Cephalalgia 30:207-213

16. Straube A, Aicher B, Förderreuther S et al (2013) Period prevalence of self-reported headache in the general population in Germany from 1995-2005 and 2009: results from annual nationwide population-based cross-sectional surveys. J Headache Pain 14:11

17. Wainscott G, Volans G, Wilkinson M (1974) Letter: Ergotamine-induced headaches. Br Med J 2:724

18. Yang CP, Chang MH, Liu PE et al (2011) Acupuncture versus topiramate in chronic migraine prophylaxis: a randomized clinical trial. Cephalalgia; 31:1510-1521

19. Yoon MS, Katsarava Z, Obermann M et al (2012) Prevalence of primary headaches in Germany: results of the German Headache Consortium Study. $J$ Headache Pain 13:215-223

20. Zeeberg P, Olesen J, Jensen R (2006) Probable medication-overuse headache: the effect of a 2-month drug-free period. Neurology 66:18941898
Der Schmerz ist sowohl im Bereich der praktisch angewandten wie auch der wissenschaftlichen Schmerzmedizin eine feste Größe. Unter dem Dach der Fachgesellschaften und unter Leitung der Herausgeber ist dies sicherlich den zahlreichen engagierten Autorinnen und Autoren sowie Ihnen, liebe Leserinnen und Leser, zu verdanken.

Maßgeblich für die Qualität und Objektivität der Beiträge sind auch die vielen qualifizierten Gutachter, die im Rahmen des Peer-Review-Prozesses nicht nur eingereichte Manuskripte inhaltlich-wissenschaftlich prüfen, sondern auch Anregungen und Empfehlungen zur konkreten Verbesserung äußern. Ihnen möchte die Schriftleitung von Der Schmerz und die Redaktion herzlich für Ihre Arbeit im Jahr 2013 danken.

- B. Arnold, Dachau
- K. Bernardy, Bochum
- J. Blunk, Köln
- H.-R. Casser, Mainz
- K.-S. Delank, Halle (Saale)
- C. Derra, Bad Mergentheim
-W. Diemer, Herne
- J. Dietrich, Bochum
- M. Dusch, Mannheim
-P. Fehrenbach, Fulda
-B. Fittkau-Tönnesmann, München
-S. Förderreuther, München
-M. Frühwald, Augsburg
-J. Gärtner, Freiburg
- H.-H. Gockel, München
-T. Graf-Baumann, Teningen
- S. Grond, Detmold
- H. Handwerker, Erlangen
- M. Hasenbring, Bochum
-W. Häuser, Saarbrücken
-F. Hoffmann, Bremen
-J. Hülsebusch, Bochum
- R. Klinger, Hamburg
-A. Koch, Aachen
-T. Kohlmann, Greifswald
- A. Kopf, Berlin
-W. Koppert, Hannover
- A. Koulousakis, Köln
-B. Kröner-Herwig, Göttingen
- N. Krumm, Aachen

- C. Lahmann, München

-H. Laubenthal, Bochum

-S. Lautenbacher, Bamberg

- G. Lindena, Kleinmachnow

-E. A. Lux, Lünen

- C. Maier, Bochum

- U. Marschall, Wuppertal

- A. Merk, Aachen

- G. Müller-Mundt, Hannover

-T. Ostermann, Herdecke

- C. Ostgathe, Erlangen

- F. Petzke, Göttingen

- R. Pothmann, Hamburg

- J. Rothaug, Jena

- R. Sabatowski, Dresden

- M. Schäfer, Berlin

- J. Schiefer, Aachen

-M. Schiltenwolf, Heidelberg

- M. Schmitter, Heidelberg

- S. Schuh-Hofer, Mannheim

- U. Schuler, Dresden

- C. Sommer, Würzburg

- H. Sorgatz, Darmstadt

- U. Stamer, Bern

-S. Stiel, Erlangen

-A. Straube, München

- K. Thieme, Marburg

-P. Thürmann, Witten/Herdecke

- M. Tingart, Aachen

- H. Traue, Ulm

- U. Wedding, Jena

-C. Wiese, Regensburg 